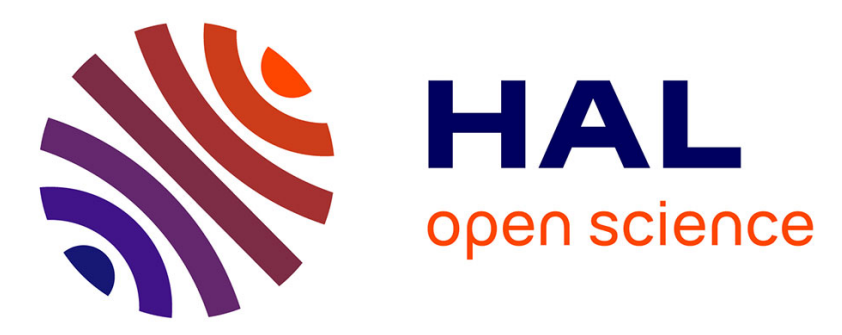

\title{
Unexpected neurological sequelae following propofol anesthesia in infants: Three case reports
}

Pierre Meyer, C. Langlois, S Soëte, J Leydet, B. Echenne, Francois Rivier, A. Bonafé, A. Roubertie

\section{- To cite this version:}

Pierre Meyer, C. Langlois, S Soëte, J Leydet, B. Echenne, et al.. Unexpected neurological sequelae following propofol anesthesia in infants: Three case reports. Brain and Development, 2010, 10.1016/j.braindev.2009.11.011 . hal-02544549

\section{HAL Id: hal-02544549 \\ https://hal.umontpellier.fr/hal-02544549}

Submitted on 16 Apr 2020

HAL is a multi-disciplinary open access archive for the deposit and dissemination of scientific research documents, whether they are published or not. The documents may come from teaching and research institutions in France or abroad, or from public or private research centers.
L'archive ouverte pluridisciplinaire HAL, est destinée au dépôt et à la diffusion de documents scientifiques de niveau recherche, publiés ou non, émanant des établissements d'enseignement et de recherche français ou étrangers, des laboratoires publics ou privés. 


\title{
Unexpected neurological sequelae following propofol anesthesia in infants: Three case reports
}

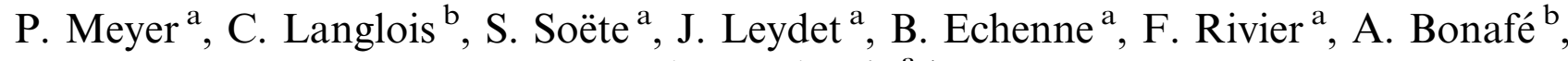 \\ A. Roubertie ${ }^{\mathrm{a}, *}$ \\ ${ }^{a}$ CHU Montpellier, Service de Neuropédiatrie, Hôpital Gui de Chauliac, Montpellier F-34000, France \\ ${ }^{\mathrm{b}} \mathrm{CHU}$ Montpellier, Service de Neuroradiologie, Hôpital Gui de Chauliac, Montpellier F-34000, France
}

\begin{abstract}
Propofol is a widely used hypnotic agent for induction and maintenance of pediatric anesthesia with a well known safety profile. Experimental in vitro studies suggest that propofol may be toxic to developing neurons. We report the cases of three infants who underwent surgery before 2 months of age for different benign pathologies. Propofol was used for induction and maintenance of anesthesia in all cases. The three patients developed convulsions with similar clinical characteristics (cluster of recurrent clinical and subclinical seizures) between the 23th and 30th hours following anesthesia. Clinical and electroencephalographic improvement was obtained between the third and fourth day of management in pediatric intensive care unit. The seizures never recurred, and the three patients underwent further uneventful general anesthesia without propofol. Follow-up of the three patients disclosed unexpected neurological dysfunction: progressive microcephaly (head circumferences were normal at birth), developmental impairment with cognitive and behavioural disturbances in two cases, and bilateral symmetrical white-matter abnormalities on cerebral magnetic resonance imaging. Conclusion: The causal relationship between propofol anesthesia and the neurological symptoms of our patients remains difficult to ascertain, but we believe that pediatricians, anesthetists and intensive care-givers should be aware of this possible adverse reaction that has never been described before.
\end{abstract}

Keywords: Anesthesia; Developmental disabilities; Microcephaly; Propofol; Seizures

\section{Introduction}

Propofol is an intravenous hypnotic who acts as a $\gamma$-aminobutyric acid. Its rapid onset of action with doserelated hypnotic effect and rapid recovery make it widely used in induction and maintenance of pediatric anesthe-

Abbreviations: EEG, electroencephalogram; MRI, magnetic resonance imaging; PICU, pediatric intensive care unit; CSF, cortico-spinal fluid; T2W, T2-weighted.

* Corresponding author. Address: Service de Neuropédiatrie, Hôpital Gui de Chauliac, 800 Avenue Fliche, 34295 Montpellier Cedex 5, France. Tel.: +334673374 22; fax: +33467337733.

E-mail address: a-roubertie@chu-montpellier.fr (A. Roubertie). sia. Despite its remarkable safety profile, there is growing interest concerning side effects of propofol in pediatric population [1], especially the propofol infusion syndrome [2] which led to its contraindication in pediatric intensive care sedation [3]. We report the case of three infants born between 2003 and 2004 who presented a cluster of recurrent seizures following propofol anesthesia, and who developed progressive unexpected neurological impairment with microcephaly.

\section{Case report}

Clinical data and further details regarding the surgical procedure are summarized in Table 1 . 


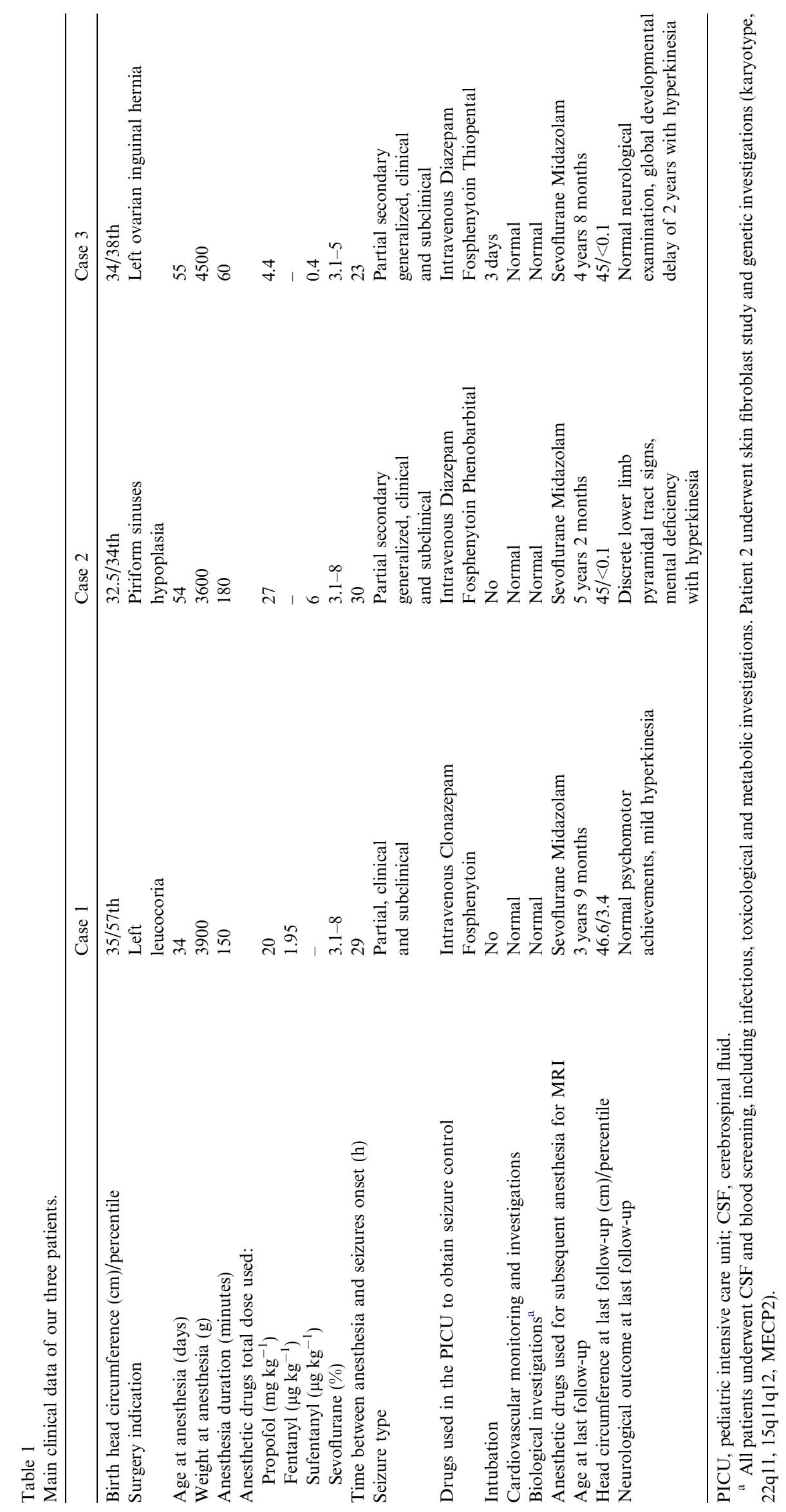




\subsection{Case 1}

This girl had a left leucocoria at birth. She is the first child of non-consanguineous Caucasian parents without medical history. She was born after a normal pregnancy, neonatal parameters were normal. A first general anesthesia was performed at 13 days of life under sevoflurane for ocular ultrasonographic and ophtalmoscopic examinations, which led to the diagnosis of isolated left congenital cataract.

At 34 days of life, an extra-capsular extraction of the crystalline lens with capsulotomy and insertion of a crystalline implant were carried under general anesthesia. The drugs used for induction and maintenance of anesthesia included propofol. Surgery and anesthesia were uneventful.

Twenty-nine hours after the intervention, clonic movements of the left upper limb with somnolence were noticed. Clinical seizures were recorded by electroencephalogram (EEG), which showed independently right-sided or left-sided onset of seizures with or without generalization and multifocal interictal spikes. Seizures were controlled after 3 days of management in Pediatric Intensive Care Unit (PICU). The patient was discharged with phenobarbital treatment.

Seizure did not recur, and the anti-epileptic treatment was stopped when the girl was 8 months of age. The child underwent four cerebral magnetic resonance imaging (MRI) under general anesthesia (without propofol) between 41 days and 3 years of age which demonstrated progressive bilateral and symmetrical fronto-parietal cortico-subcortical involvement (Fig. 1a and b). Anesthetic procedure was uneventful.

This girl managed to walk unaided and emitted her first words before one year of age. First sentences were produced at 3 years. At 38 months, she was described as a very active girl, but her behavior was otherwise normal, and her educational achievements were concordant with her age. Neurological examination was normal. The most outstanding clinical finding in her follow-up was a progressive microcephaly around the third percentile for a normal head circumference at birth (Fig. 2).

\subsection{Case 2}

This girl had feeding difficulties from the first days of life, leading to the diagnosis of piriform sinuses hypoplasia. She is the second child of non-consanguineous Caucasian parents without medical history. The girl was born at 34 weeks of gestation after premature spontaneous labor. Neonatal parameters were normal.

The surgical treatment of piriform sinuses hypoplasia under general anesthesia was performed at 54 days of life. The drugs used for induction and maintenance of anesthesia included propofol. Surgery and anesthesia were uneventful.
Thirty hours after the anesthesia, the patient presented clonic movements of the right upper extremity, followed by clusters of clonic seizures, initially on the left side of the body, then involving both sides. The EEG recorded a status epilepticus characterized by recurrent seizures with multifocal sites of onset independently on both hemispheres (right anterior and left temporal regions). Clinical and subclinical seizures disappeared after 4 days of management in PICU; the patient was discharged with phenobarbital treatment. A brain MRI was normal 7 days after the surgery.

The girl underwent two MRI under general anesthesia (without propofol) at 13 and 38 months of age which demonstrated bilateral and symmetrical cortico-subcortical parieto-occipital and cortical cerebellar involvements (Fig. 1c and d).

The clinical seizures did not recur, but due to the persistence of intercritical epileptic abnormalities on the EEG, phenobarbital was substituted by vigabatrin when the patient was 8 months of age, and stopped ten months later.

The neurological follow-up revealed a progressive and severe microcephaly far below the third percentile (Fig. 2). The girl managed to walk unaided at 14 months, but language was delayed (first short phrases at 37 months of age), and behavior was characterized by hyperactivity and attention deficiency. Clinical examination disclosed fine motor skills disabilities, discrete pyramidal tract signs on the lower limbs with a distal spasticity. At 4 years of age, WPPSI-III evaluation (Wechsler Preschool and Primary Scale of Intelligence) confirmed the cognitive deficiency with heterogeneous capacities (Verbal Intellectual Quotient: 76; Performance Intellectual Quotient: 46). This patient has a special educational assistance in a normal school.

\subsection{Case 3}

This girl is the second child of non-consanguineous Caucasian parents. Her father and brother are affected by Bessel Hagen disease (multiple osteochondromas). She was born after a normal pregnancy at 36 weeks of gestation, neonatal parameters were normal. The patient had a normal development up to 50 days of life when she was referred for a left ovarian inguinal hernia.

She underwent a surgical herniotomy under general anesthesia at 55 days of life. The drugs used for induction and maintenance of anesthesia included propofol. Surgery and anesthesia were uneventful.

Twenty-three hours after the anesthetic induction the patient presented recurrent seizures with right-sided head deviation and clonic jerks of the upper extremities, followed by generalized clonic movements. Clinical seizures were recorded by EEG which showed asynchronous bitemporal slow spikes, and right parietal spikes and polyspikes. The seizures were controlled after 3 days of man- 
a

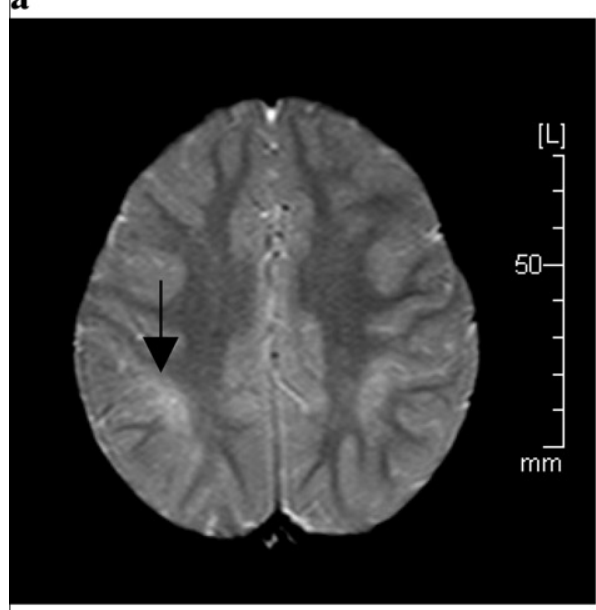

c

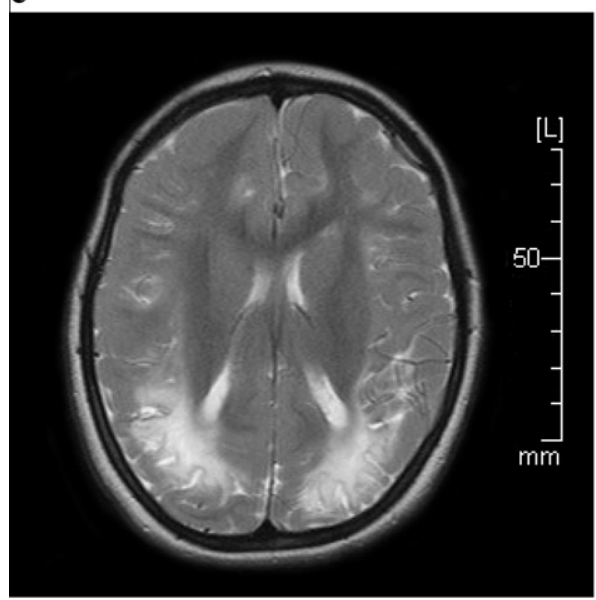

e

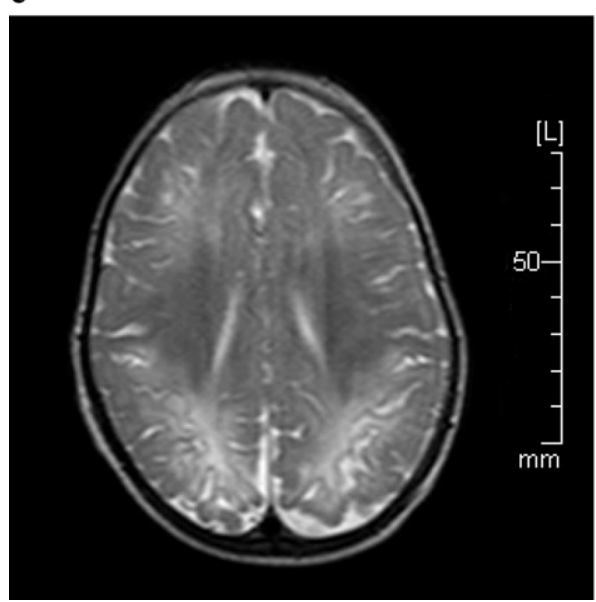

b

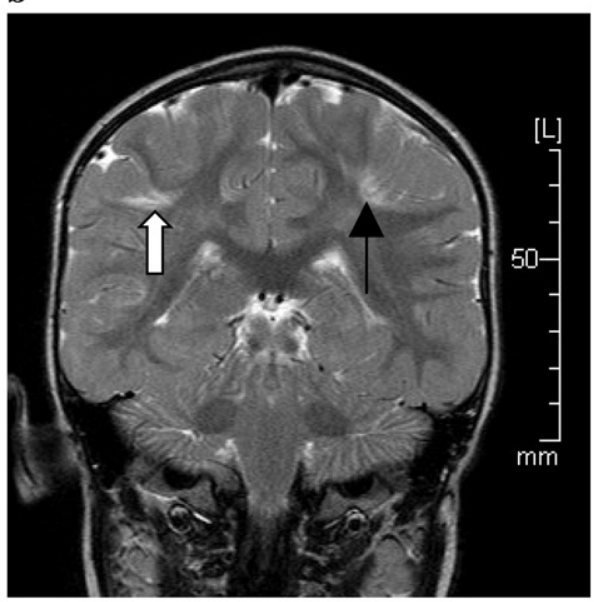

d

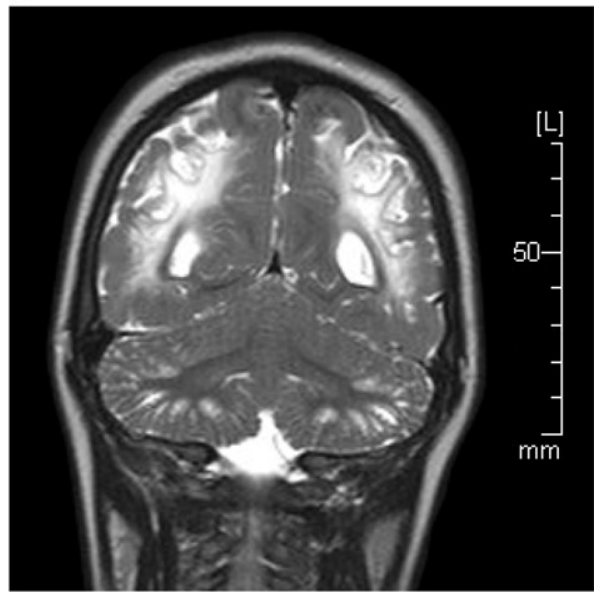

f

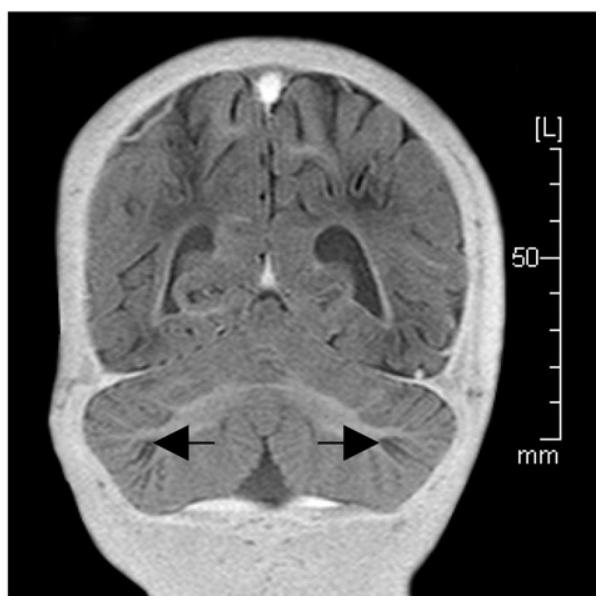

Fig. 1. (a and b) Case 1, brain MRI performed at 2 years 5 months. T2-weighted axial (a) and coronal (b) images demonstrate bilateral and symmetrical hyper T2 signal at the bottom of the sulci of the parieto-frontal cortex (black arrow). The adjacent sub-cortical white matter is slightly involved (white arrow). (c and d) Case 2, brain MRI performed at 3 years 5 months. T2-weighted axial (c) and coronal (d) images demonstrate bilateral and symmetrical increased signal of the deep periventricular and sub-cortical white matter with involvement of the adjacent cortex at the bottom of the sulci of the parieto-occipital areas. Posterior fossa images (d) demonstrate bilateral and symmetrical involvement of the cerebellar cortex without involvement of the white matter. (e and f) Case 3, brain MRI performed at 1 year 7 months. T2-weighted axial images (e) demonstrate bilateral and symmetrical hypersignal of the deep and sub-cortical white matter and of the adjacent cortex in the fronto-parietal regions. Coronal $\mathrm{T} 1$ inversion-recovery images (f) demonstrate bilateral symmetrical hyposignal of the cerebellar cortex with normal cerebellar white matter (black arrow). 

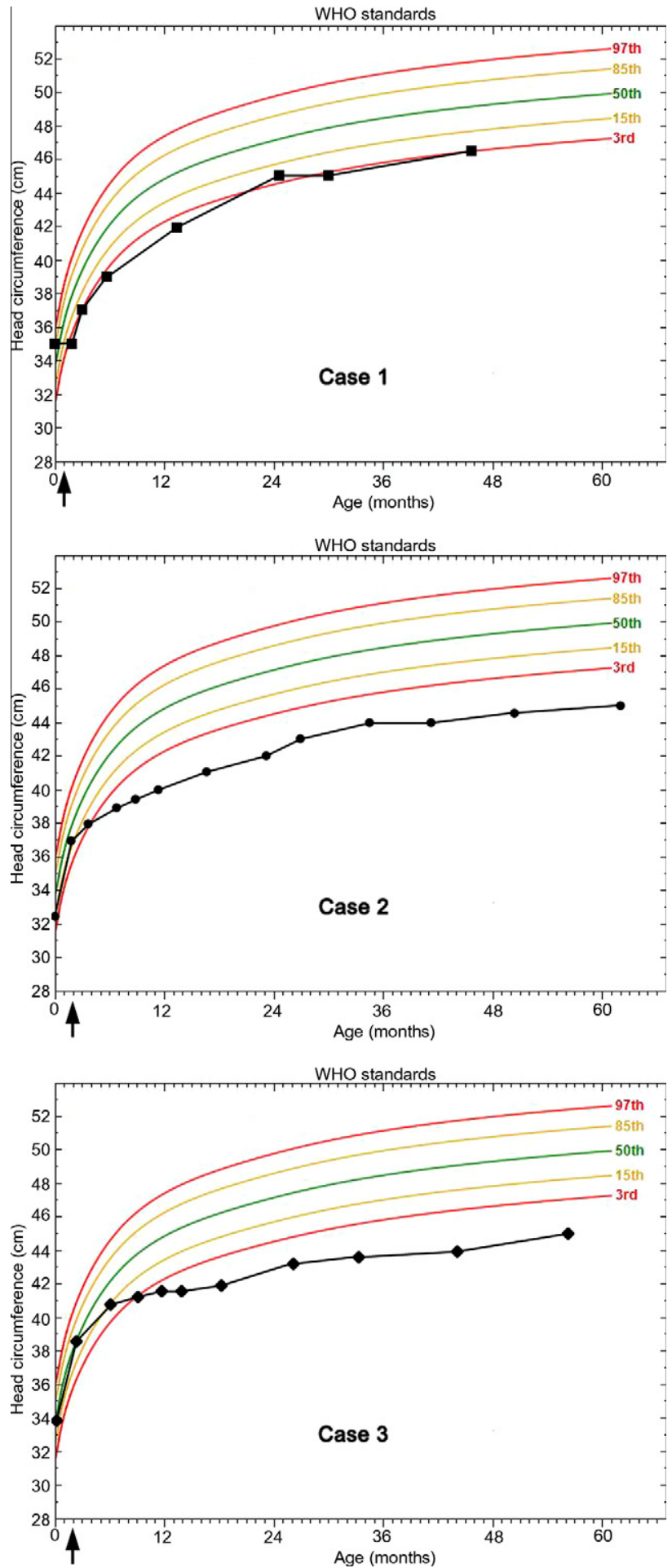

Fig. 2. Head circumference growth of the three patients, based on WHO standards. Occurrence of propofol anesthesia is indicated by the black arrow.

agement in PICU. Transfontanellar ultrasonography and cerebral tomodensitometry were normal. The patient was discharged with phenobarbital treatment.

The parents initially refused early cerebral MRI under general anesthesia. This examination was carried out (without propofol) at the age of 19 months and demonstrated bilateral and symmetrical cortico-subcortical fronto-parietal and cortical cerebellar involvement (Fig. 1e and f).

The neurological follow-up revealed a progressive and severe microcephaly far below the third percentile (Fig. 2). Motor development was normal (walk unaided at 14 months), but language was markedly delayed (short sentences at 4.5 years); hyperkinesia and attention deficiency were noticed early in life, and were very disabling. Clinical examination disclosed poor fine motor skills with praxis disorders. At 4 years, the girl did not manage to complete all the subtests of WPPSI-III scale; her estimated level was concordant with 2.5 years. This patient has a special educational assistance in a normal school.

\section{Discussion}

Clinical characteristics of our patients are very similar: surgery during the second month of life for benign pathology, time-limited cluster of seizures occurring between the 23rd and 30th hours after propofol anesthesia, initial good rapid recovery, progressive microcephaly, neurodevelopmental delay in two out of three patients and brain MRI bilateral symmetrical cortico-sub-cortical abnormalities involving mainly the parietal areas.

Propofol is a widely used anesthesic agent, which may be given to about 2 million patients per year in France alone [4]. The proconvulsivant effect of propofol has been largely described in the literature. Propofol infusion may increase the frequency of spikes during electrocorticography in certain patients [5]; in some cases, it has been demonstrated that neocortical foci with epileptiform signs might be activated 20-30 s after a bolus administration of propofol [6]. Moreover, seizures and seizure-like phenomena (SLP) following propofol use have been well reported, leading to a warning about its use in patients with epilepsy [7]. Through a large literature analysis, Walder et al. reported SLP in 70 patients from 1 month to 82 years without epilepsia: SLP were delayed from 33 min to 6 days following anesthesia in 16 patients ( $23 \%)$; unfortunately, no data are available concerning the follow-up of the patients [4]. The time of occurrence of seizure in our patients (i.e. between the 23rd and 30th hours after propofol anesthesia) definitely fits with what is reported by Walder et al.

On the other hand, the other anesthetic agents used for our three patients have no documented proconvulsivant effect: there are only some descriptions of tonic-clonic movements following fentanyl or sufentanyl administration, and there are many reports of EEG recording during fentanyl anesthesia without evidence of epileptiform activity [8]. The use of sevoflurane may be associated with cortical epileptiform EEG signs, usually without clinical manifestation [9] and we found only two adult case reports of SLP during recovery of sevo- 
flurane anesthesia [10,11]. Furthermore, all our patients received sevoflurane without side effects during MRI performed some months after the acute epileptic episode, and the subsequent anesthetic procedures performed without propofol were uneventful.

Laboratory tests and close review of the per-anesthetic and PICU charts of our three patients did not disclose any evidence of drug overdose (in particular propofol dose was correct according to the weight, i.e. $2.5 \mathrm{mg} \mathrm{kg}^{-1}$ for induction and $199 \mu \mathrm{g} \mathrm{kg}^{-1} \mathrm{~min}^{-1}$ for maintenance of anesthesia [12]). No sign of cardiovascular instability (during or after the surgical procedure) nor hypoglycemia were reported, and no sign of propofol infusion syndrome were diagnosed (no metabolic acidosis, rhabdomyolysis, hyperlipidemia, and enlarged or fatty liver) [13]. Moreover, metabolic or genetic studies and follow-up of our patients raised no evidence for underlying metabolic or genetic disorder with seizure susceptibility.

Altogether, these data provide consistent clues for the potential imputability of propofol as the main causing agent of the occurrence of seizures in our three patients.

The three patients showed irreversible similar bilateral and symmetrical posterior white matter and cortical abnormalities on brain MRI (Fig. 1). The abnormalities were expanded into multiple arterials territories and not only in the watershed junction; the cerebellar cortex was focally involved (case 2 and 3). Unfortunately, diffusion images and spectroscopy were not performed in daily patient MRI management at the time of our three patients' referrals. The diagnosis of Posterior Reversible Encephalopathy Syndrome (PRES) seems unlikely as blood pressure monitoring of our three infants during and postoperative states has always been normal, and cerebral lesions were not reversible; moreover, PRES has not been reported up to now in infants younger than 2 years of age, and has never been described after propofol use [14]. The brain abnormalities observed on the MRI have an "ulegyric pattern" but the medical history our three patients is devoid of any equivocal asphyxic event (especially the monitoring during surgery and recovering was definitely uneventful) [15].

There is a striking analogy between the topography of the brain damage in our patients, and the study of Juengling et al.; actually, significant dose-related hypometabolic areas were found in the parieto-occipital cortex bilaterally using fluorodeoxyglucose positron emission tomography in epileptic children during propofol-induced sedation, after comparison with both healthy adult control group and an age-matched child intra-group control [16]. Thus, one can hypothesize that the white-matter abnormalities seen in our patients may be secondary to propofol administration.

The three patients had normal head circumference at birth but subsequently developed microcephaly around the 3rd percentile in one case and far below the 3rd per- centile (Fig. 2) with neurodevelopmental impairment in two cases.

Some in vitro studies have shown that propofol may cause irreversible lesions to GABAergic neurons and glial cells when given during brain development $[17,18]$, longterm changes in rat immature dendritic arbor development [19], and chick-embryonic growth cone collapse and neurite destruction [20]. All this experimental data suggests that propofol is toxic to developing neurons and suppresses their growth. Up to now, microcephaly has never been reported clinically or experimentally, in animals or humans, after propofol administration. One might suspect that the young age of our patients (i.e. during neuronal development) might be implicated in the type of symptoms (clusters of seizures), the topography of the lesions and the neurological sequelae, involving vascular and/or toxic mechanism.

\section{Conclusion}

For these three infants, the definite causal relationship between propofol and the neurological sequelae (microcephaly, developmental impairment with cognitive and behavioral disturbances, and bilateral corticosubcortical ulegyric-like abnormalities) remains difficult to ascertain, and the epidemiologic value of such case reports is poor, but we believe that pediatricians, radiologists, anesthetists and intensive care-givers should be aware of this possible adverse reaction that has never been described before.

\section{References}

[1] Mellon RD, Simone AF, Rappaport BA. Use of anesthetic agents in neonates and young children. Anesth Analg 2007;104:509-20.

[2] Okamoto MP, Kawaguchi DL, Amin AN. Evaluation of propofol infusion syndrome in pediatric intensive care. Am J Health Syst Pharm 2003;60:2007-14.

[3] U.S. Food and Drug Administration. Medical product safety labeling changes approved by FDA center for drug evaluation and research 2007 Feb. Available from URL: www.fda.gov/ medwatch/safety/2007/Feb_PI/Diprivan_PI.pdf.

[4] Walder B, Tramèr MR, Seeck M. Seizure-like phenomena and propofol: a systematic review. Neurology 2002;58:1327-32.

[5] Hufnagel A, Elger CE, Nadstawek J, Stoeckel H, Böker DK. Specific response of the epileptic focus to anesthesia with propofol. J Epilepsy 1990;3:37-45.

[6] Hodkinson BP, Frith RW, Mee EW. Propofol and the electroencephalogram. Lancet 1987;2:1518.

[7] Walker G. Disoprivan 1\%. ABPI compendium of data sheets and summaries of product characteristics. Datapharm Publications: London; 1998, p. 1093-4.

[8] Bruder N, Bonnet M. Epileptogenic drugs in anesthesia. Ann Fr Anesth Reanim 2001;20:171-9.

[9] Constant I, Seeman R, Murat I. Sevoflurane and epileptiform EEG changes. Paediatr Anaesth 2005;15:266-74.

[10] Hilty CA, Drummond JC. Seizure-like activity on emergence from sevoflurane anesthesia. Anesthesiology 2000;93:1357-9.

[11] Terasako K, Ishii S. Postoperative seizure-like activity following sevoflurane anesthesia. Acta Anaesthesiol Scand 1996;40:953-4. 
[12] U.S. Food and Drug Administration. Center for drug evaluation and research. Pediatric exclusivity labeling changes as of June 24, 2008. Available from URL: http://www.fda.gov/cder/pediatric/ labelchange.htm.

[13] Kam PC, Cardone D. Propofol infusion syndrome. Anaesthesia 2007;62:690-701.

[14] Yokobori S, Yokota H, Yamamoto Y. Pediatric posterior reversible leukoencephalopathy syndrome and NSAID-induced acute tubular interstitial nephritis. Pediatr Neurol 2006;34:245-7.

[15] Villani F, D'Incerti L, Granata T, Battaglia G, Vitali P, Chiapparini L, et al. Epileptic and imaging findings in perinatal hypoxic-ischemic encephalopathy with ulegyria. Epilepsy Res 2003;55:235-43

[16] Juengling FD, Kassubek J, Martens Le Bouar H, Reinhardt MJ, Krause T, Nitzsche EU, et al. Cerebral regional hypometabolism caused by propofol-induced sedation in children with severe myoclonic epilepsy: a study using fluorodeoxyglucose positron emission tomography and statistical parametric mapping. Neurosci Lett 2002;335:79-82.

[17] Honegger P, Matthieu JM. Selective toxicity of the general anesthetic propofol for GABAergic neurons in rat brain cell cultures. J Neurosci Res 1996;45:631-6.

[18] Spahr-Schopfer I, Vutskits L, Toni N, Buchs PA, Parisi L, Muller D. Differential neurotoxic effects of propofol on dissociated cortical cells and organotypic hippocampal cultures. Anesthesiology 2000;92:1408-17.

[19] Vutskits L, Gascon E, Tassonyi E, Kiss JZ. Clinically relevant concentrations of propofol but not midazolam alter in vitro dendritic development of isolated gamma-aminobutyric acidpositive interneurons. Anesthesiology 2005;102:970-6.

[20] Al-Jahdari WS, Saito S, Nakano T, Goto F. Propofol induces growth cone collapse and neurite retractions in chick explant culture. Can J Anesth 2006;53:1078-85. 